\title{
The spore coat of the bean anthracnose fungus Colletotrichum lindemuthianum is required for adhesion, appressorium development and pathogenicity
}

\author{
Sarah L. Rawlings ${ }^{\mathrm{a}}$, Richard J. O'Connell ${ }^{\mathrm{b}, 1}$, Jonathan R. Green ${ }^{\mathrm{a}, *}$ \\ ${ }^{a}$ School of Biosciences, University of Birmingham, Birmingham B15 2TT, UK \\ ${ }^{\mathrm{b}}$ IACR-Long Ashton Research Station, Long Ashton, Bristol BS41 9AF, UK
}

Accepted 24 July 2007

\begin{abstract}
The spores (conidia) of the bean anthracnose fungal pathogen, Colletotrichum lindemuthianum, adhere to the aerial parts of plants to initiate the infection process. In previous studies we have shown that the Colletotrichum spores are surrounded by a fibrillar spore coat, comprising several major glycoproteins. Previous evidence showed that a monoclonal antibody (UB20) that recognised these glycoproteins was able to inhibit adhesion of spores to a hydrophobic surface. In this paper we have further studied the role of the spore coat in adhesion, germination and fungal development by studying the effects of UB20 and protease treatment of spores. The latter treatment has previously been shown to remove the spore coat. Spores germinate on glass, polystyrene and water agar, however, appressoria only develop on glass or polystyrene, showing a requirement for a hard surface. Removal of the spore coat with protease inhibits adhesion at $30 \mathrm{~min}$, before the secretion of ECM glycoproteins. Protease treatment also inhibits the development of appressoria and reduces pathogenicity on leaves. Incubation of spores with the MAb UB20 inhibits adhesion at 30 min, but does not affect appressorium formation or pathogenicity. The results suggest that an intact spore coat has two functions; it is required for adhesion to a hydrophobic surface and for the detection of a hard surface necessary for appressorium formation. We suggest that contact with a hard surface, rather than adhesion, is the key event leading to appressorium formation.
\end{abstract}

(C) 2007 Elsevier Ltd. All rights reserved.

Keywords: Adhesion; Appressorium; Bean; Colletotrichum lindemuthianum; Conidia; Extracellular matrix; Spore; Spore coat

\section{Introduction}

Interactions of spores of fungal plant pathogens with a substratum can be important for germination, appressorium formation and efficient infection of susceptible hosts. Spore contact with a hard surface has been shown to be important for germination and/or appressorium formation for many fungal pathogens that infect the aerial parts of susceptible plants, e.g. the rice blast fungus Magnaporthe grisea [1], Botrytis cinerea [2] and the anthracnose

\footnotetext{
*Corresponding author. Tel.: + 44121414 5574; fax: + 441214145925.

E-mail address: j.r.green@bham.ac.uk (J.R. Green).

${ }^{1}$ Present address: Department of Plant Microbe Interactions, Max Planck Institute for Plant Breeding Research, Carl-von-Linné-Weg 10, 50829 Köln, Germany.
}

fungi Colletotrichum gloeosporioides [3,4], C. trifolii [5], C. coccodes and C. dermatium [3]. Whether the surface is hydrophilic or hydrophobic can also affect germination and/or appressorium formation, e.g. C. lagenarium germinates more effectively on hydrophilic surfaces [6], whereas C. graminicola and Phyllosticta amelicida germinate and produces appressoria preferentially on hydrophobic surfaces [7-9]. In some cases, chemical cues are also required for appressorium formation e.g. the avocado pathogen, C. gloeosporioides requires ethylene and avocado wax to trigger appressorium formation, but these are only effective after the spores have been in contact with a hard surface for $2 \mathrm{~h}[4,10]$. Studies on Colletotrichum species and $M$. grisea have shown that genes encoding proteins involved in cell signalling, e.g. calmodulin, protein kinase, CAMKinase, MAP kinases as well as other functions, 
including ubiquitination, are switched on shortly after spore contact with a hard surface [5,11-15]. Other studies have shown that specific signalling pathways are involved in germination and appressorium formation after contact of spores with a hard surface $[2,3]$.

Spore adhesion to the substratum can provide the spore with stability and time to receive stimuli required to initiate the signal transduction pathways essential for germination and appressorium formation. Adhesion has been implicated as being required for spore germination (e.g. Phyllosticta ampelicida and other Phyllosticta species [16,17], C. graminicola $[7,8]$ and $C$. trifolii [18]) and appressorium development (e.g. M. grisea [19]; C. graminicola $[7,8])$. It has also been shown that adhesion of $C$. graminicola spores is required for efficient infection of corn leaves [20]. Disruption of the adhesion of fungal spores to leaves can lead to them becoming dislodged by environmental factors such as wind and rain.

The surface features of fungal spores and germ-tubes that mediate the detection of a hard surface and adhesion have not been fully characterised in any system. Studies on spore adhesion in several Colletotrichum species suggest that both passive and active processes are involved. In C. graminicola and C. musae, initial adhesion involves preformed proteins at the spore surface followed by synthesis and secretion of adhesive proteins at the tip of the conidium, which are released before germination [21-23]. For Blumeria graminis, a conidial ECM is rapidly released after spore contact with the substratum and this is an active process [24,25]. For $M$. grisea, a pre-formed adhesive is released upon spore contact with a surface, then ECM glycoproteins are secreted which consolidate adhesion of germ-tubes and appressoria [26]. A gene encoding a putative ECM protein, EMP1, has been identified in $M$. grisea [27]. Zoospores of the Oomycete pathogen, Phytophthora cinnamomi, release an adhesive with many copies of a motif found in adhesins of animals and malarial parasites [28]. Some fungal spores have a spore coat or distinct layer of material outside of the spore wall. In Phyllosticta ampelicida this has been implicated as having a role in adhesion and spore germination [16] and in Discula umbrinella the spore coat has been implicated in adhesion [29].

Many studies on the adhesion and signalling processes outlined above have utilised fungi of the genus Colletotrichum, which are important and successful plant pathogens causing both pre- and post-harvest infections in temperate and tropical environments [30,31]. We have used C. lindemuthianum, the hemibiotrophic bean anthracnose fungus, for our studies. Using transmission electron microscopy (TEM), spores of $C$. lindemuthianum have been shown to possess a carbohydrate-rich, fibrillar spore coat which is not found on either germ-tubes or appressoria. The spore coat is composed of densely packed fibres arranged perpendicularly to the cell wall, and possesses a number of irregularly shaped pores giving it a honey-comb like appearance [31,32]. Similar structures have also been observed in P. ampellicida [9], C. truncatum [33] and D. umbrinella [29]. In previous studies, we found evidence showing that the adhesion of $C$. lindemuthianum spores involves pre-formed glycoproteins that are present in the specialised spore coat. The spore coat was removed from spores using hot water and SDS treatments, and analysis by SDS-PAGE showed that the spore coat was composed of relatively few glycoproteins, with a major component at $110 \mathrm{kDa}$. A monoclonal antibody (MAb), UB20, that binds primarily to spores [34] and to this major glycoprotein, was able to inhibit the adhesion of spores to polystyrene dishes [35] in an adhesion assay that was adapted from that used by Mercure et al. [22]. In addition, polystyrene beads were shown to bind to the $110 \mathrm{kDa}$ glycoprotein on Western blots [35]. Overall, the evidence suggested a role for the spore coat in the initial stages of C. lindemuthianum spore adhesion to hydrophobic surfaces.

After spore germination, the germ-tubes and appressoria of $C$. lindemuthianum are surrounded by an extracellular matrix (ECM) of secreted glycoproteins, some of which have been identified with MAbs UB26 and UB31 $[32,36,37]$. These antibodies label footprints of ECM material remaining on glass slides after removal of adherent cells by sonication, suggesting that they recognise adhesive components.

As described above, in previous studies we established that incubation of $C$. lindemuthianum spores with the $\mathrm{MAb}$ UB20 inhibited adhesion to a hydrophobic surface [35]. The aims of the studies reported in this paper were to determine the role of the spore coat in germination, appressorium formation and pathogenicity of this fungus and to determine the relationship between spore adhesion and these processes. This was achieved by examining the effects of UB20 or protease treatment of spores in a variety of assays. In addition, we have explored the importance of spore interactions with a hard surface leading to appressorium formation, and the role of the spore coat. The results suggest that the spore coat of $C$. lindemuthianum has at least two functions; it is necessary for adhesion of spores to a hydrophobic surface and it is required for interactions with a hard surface to initiate the differentiation of appressoria. However, the spore coat is not required for spore germination to occur, and the adhesion of spores is not required for subsequent appressorium formation; contact with a hard surface is the key event required for the latter to occur.

\section{Materials and methods}

\subsection{Fungal culture and preparation of washed spores}

Race $\kappa$ (Long Ashton Research Station (LARS) culture no. 137) and race $\gamma$ (ATCC 56987; LARS 129) cultures of C. lindemuthianum (Sacc. and Magn.) Briosi \& Cav. were grown on a medium described by Mathur et al. [38] and maintained as described previously [34]. Spores were obtained from 7 day-old cultures by washing with $50 \mathrm{ml}$ 
of distilled water $\left(\mathrm{dH}_{2} \mathrm{O}\right)$. The removal of the water-soluble acervular mucilage from spores [39] was achieved by centrifuging twice at $1200 \mathrm{~g}$, for $10 \mathrm{~min}$ at room temperature $\left(18^{\circ} \mathrm{C}\right)$. Spore surface extracts were prepared by resuspending washed spores (approx. $1 \times 10^{8}$ ) in $250 \mu 1$ deionised water and heating to $90{ }^{\circ} \mathrm{C}$ for $10 \mathrm{~min}$ [35].

\subsection{Monoclonal antibodies}

MAbs used in indirect immunofluorescence (IIF) experiments (see below) were in the form of tissue culture supernatants (TCS) produced by growing mouse hybridoma cell lines. MAbs UB20 [34], UB26 and UB31 [32,37] were raised against different fungal infection structures. MAb UB20 binds to the spore coat of $C$. lindemuthianum [34,35], whereas UB26 and UB31 bind to glycoproteins present in the ECMs surrounding germ-tubes and appressoria $[32,34,36]$. MAb UBIM22, raised against rat bone cells [40], does not cross-react with fungal infection structures and was used as a negative control. The MAbs UB2 0 and UBIM22 are both IgG1 and were purified from TCS by precipitation with saturated ammonium sulphate, followed by dialysis and passage through a protein $\mathrm{G}$ column (Sigma, UK). The IgGs were eluted with $100 \mathrm{mM}$ glycine, $\mathrm{pH} 3$ [41].

\subsection{Indirect immunofluorescence}

Multiwell glass slides (ICN Biomedicals Ltd, High Wycombe, Bucks, UK), were cleaned with 2\% $(w / v)$ Decon 90 detergent, $5 \%(v / v)$ acetic acid and rinsed thoroughly with $\mathrm{dH}_{2} \mathrm{O}$, before silanisation by immersion in dimethyldichlorosilane in $2 \%(v / v) 1,1,1$, trichloroethane for $2 \mathrm{~min}$ (BDH/Merck Ltd, Poole, Dorset, UK). Slides were then allowed to air dry and traces of solvent were removed by heating to $180^{\circ} \mathrm{C}$ for $30 \mathrm{~min}$. Spore suspension $(50 \mu 1$ of $5 \times 10^{5}$ spores $\mathrm{ml}^{-1}$ ) was placed in the wells of these slides and incubated at $23^{\circ} \mathrm{C}$ in a humid box in the dark for various times. After incubation, the slides were rinsed once in $\mathrm{dH}_{2} \mathrm{O}$ and the spores were fixed with $4 \%(w / v)$ paraformaldehyde for $1 \mathrm{~h}$. Fungal cells were immunolabelled with MAbs followed by rabbit anti-mouse IgG antibody conjugated with fluorescein isothiocyanate (FITC-RAMIG) as described previously [34]. Slides were observed with a Zeiss Axiophot microscope using differential interference contrast and UV-epifluorescence.

\subsection{Assessment of spore germination and appressorium formation}

Spores were tested for their ability to germinate and form appressoria on different surfaces. Drops $(25 \mu \mathrm{l})$ of spore suspension $\left(5 \times 10^{5}\right.$ spores $\left.\mathrm{ml}^{-1}\right)$ were incubated on acid-washed multiwell glass slides, slides coated with $2 \%$ water agar, or polystyrene Petri dishes for $24 \mathrm{~h}$, in a humid box in the dark, at $23^{\circ} \mathrm{C}$. The samples were then observed with the $\times 25$ objective of an inverted microscope and the percentage germination and appressorial development were calculated from 10 random fields of view.

\subsection{Adhesion assay}

An adhesion assay based on a method described by Mercure et al. [22] was adapted for use with $C$. lindemuthianum spores [35]. Spores were diluted to a final concentration of $1 \times 10^{6}$ spores $\mathrm{ml}^{-1}$ with $\mathrm{dH}_{2} \mathrm{O}$. The spore suspension $(50 \mu \mathrm{l})$ was pipetted into $3 \times 5 \mathrm{~mm}^{2}$ marked areas on the base of polystyrene Petri dishes $(5 \mathrm{~cm}$ diameter, Sterilin) or multiwell glass slides (ICN). After incubating for the appropriate time at $18{ }^{\circ} \mathrm{C}, 10$ random fields of view per marked area were counted using the $\times 25$ objective on an inverted microscope. After the addition of $6 \mathrm{ml}$ of $\mathrm{dH}_{2} \mathrm{O}$ the Petri dish was rotated on an orbital shaker for $10 \mathrm{~s}$ at $500 \mathrm{rpm}$. Random fields of view were then recounted as before and the percentage adhesion calculated by dividing the number of conidia/germinated conidia that remained attached by the number that had originally settled onto the polystyrene.

To test the effect of sodium azide or cycloheximide treatment on the adhesion of spores, aliquots of spore suspension $(75 \mu \mathrm{l})$ were incubated with $75 \mu \mathrm{l}$ of varying concentrations of the compound being tested and incubated at $18{ }^{\circ} \mathrm{C}$ for $15 \mathrm{~min}$ before addition to Petri dishes as described above.

\subsection{Effects of protease treatment and MAbs on spore adhesion, germination and appressorium formation}

To test whether removal of the spore coat had any effect on adhesion, germination or appressorium formation, spores were treated with protease (Pronase E: Sigma, Poole, Dorset, UK). Spores, harvested and washed as described above, were incubated for $30 \mathrm{~min}$ at $30^{\circ} \mathrm{C}$ in $5 \mathrm{mg} \mathrm{ml}^{-1}$ protease dissolved in $10 \mathrm{mM}$ Tris- $\mathrm{HCl}, \mathrm{pH}$ 7.6. Protease denatured by heating to $95^{\circ} \mathrm{C}$ for $10 \mathrm{~min}$ was used as a control. Spores were then washed three times by centrifugation at $3000 \mathrm{~g}$ for $10 \mathrm{~min}$. The supernatant was discarded and spores were diluted to a concentration of $5 \times 10^{5}$ spores $\mathrm{ml}^{-1}$ before applying $25 \mu \mathrm{l}$ to polystyrene Petri dishes or glass slides. Adhesion, spore germination and appressorium formation were then measured as above. In preliminary experiments, spores treated with protease were washed up to five times by centrifugation, but the results obtained were similar to those obtained for spores washed three times.

To test the effects of MAbs on adhesion, germination or appressorium formation, aliquots $(25 \mu \mathrm{l})$ of spore suspension $\left(1 \times 10^{6}\right.$ spores $\left.\mathrm{ml}^{-1}\right)$ were mixed with $25 \mu \mathrm{l}$ the purified IgG (either UB20 or UBIM22, $50 \mu \mathrm{g}$ protein $\mathrm{ml}^{-1}$ ) and were pipetted into $3 \times 5 \mathrm{~mm}^{2}$ marked areas on polystyrene Petri dishes. Percentage adhesion, germination and appressorium formation were then determined as described above. 


\subsection{Pathogenicity assay}

The ability of spores to infect 10 day-old detached bean leaves was tested as follows. Spores were washed twice and diluted to a concentration of $1 \times 10^{6}$ spores $\mathrm{ml}^{-1}$. The spore suspension $(3 \mathrm{ml})$ was pipetted onto the abaxial leaf surface, spread with a soft brush, and incubated for approximately 4 days in a humid box at $17^{\circ} \mathrm{C}$. Epidermal peels were then taken to assess by light microscopy whether appressoria and intracellular hyphae had formed. To test whether MAb UB20 affected the ability of conidia to infect bean leaves, $1.5 \mathrm{ml}$ of spore suspension $\left(1 \times 10^{6}\right.$ spores $\left.\mathrm{ml}^{-1}\right)$ was incubated with $1.5 \mathrm{ml} \mathrm{UB} 20 \mathrm{IgG}$ $\left(50 \mu \mathrm{g}\right.$ protein $\left.\mathrm{ml}^{-1}\right)$, and then spread over the leaf surface. Spores pre-treated with protease and heat-denatured protease, as described above, were also tested in the leaf assay.

\section{Results}

\subsection{Spore germination and appressorium formation}

Germination and appressorium formation were assessed after $C$. lindemuthianum spores were allowed to settle onto glass (hydrophilic, hard surface), polystyrene (hydrophobic, hard surface) or water agar (hydrophilic, soft surface). The results showed that spores germinated on all these surfaces but appressoria only developed on glass or polystyrene (Table 1). Spores that germinated in liquid culture (e.g. Czapek medium) did not differentiate appressoria in any conditions tested (results not shown). These experiments, and those described below, were generally repeated at least three times and representative results have been presented.

\subsection{Characteristics of spore adhesion}

A time-course study of spore adhesion on polystyrene and glass was performed. Polystyrene has a similar hydrophobicity to bean hypocotyls [35], whereas acidwashed glass is hydrophilic. The results showed that adhesion to polystyrene was rapid and almost $100 \%$ after $10 \mathrm{~min}$, whereas on glass, adhesion after $10 \mathrm{~min}$ was low $(<20 \%)$ and gradually increased over a $2 \mathrm{~h}$ period (Fig. 1$)$. Although Colletotrichum spores are embedded in a

Table 1

Spore germination and appressorium formation on different substrata

\begin{tabular}{lll}
\hline Substratum & Germination $^{\mathrm{a}}(\%)$ & Appressoria $^{\mathrm{a}}(\%)$ \\
\hline Water agar & $50.7 \pm 4.3$ & 0 \\
Glass & $65.8 \pm 5.9$ & $50.3 \pm 5.2$ \\
Polystyrene & $62.3 \pm 5.2$ & $53.1 \pm 6.1$ \\
\hline
\end{tabular}

\footnotetext{
${ }^{\mathrm{a}}$ Germination and appressorium formation were assessed after $24 \mathrm{~h}$ incubation on each substrate. Results are the means \pm the standard errors from three replicates.
}

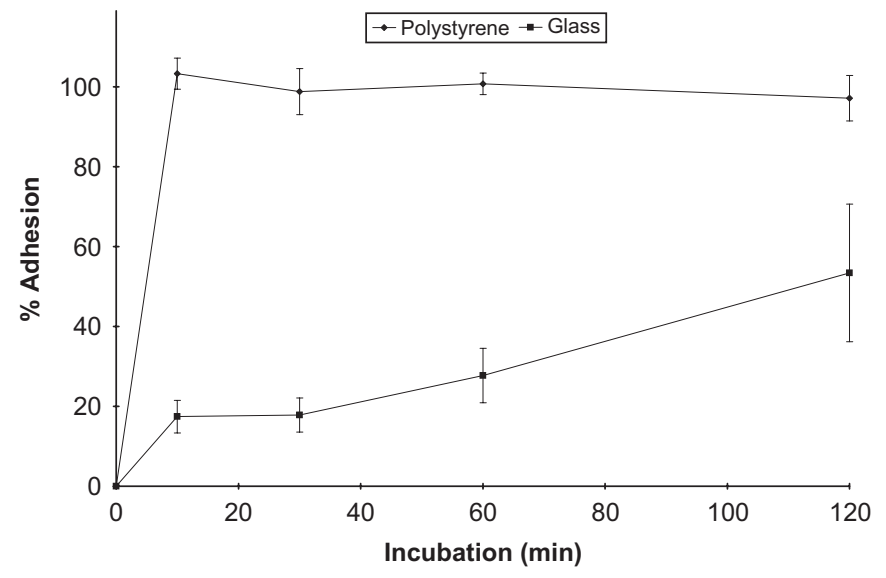

Fig. 1. Time course of spore adhesion on glass and polystyrene. Spores were incubated for various times before adhesion was determined. Error bars indicate the standard deviation of the mean of three replicates.

water-soluble mucilage which contains self-inhibitors of germination [39], this had no role in adhesion and, in fact inhibited adhesion slightly, when compared to washed spores (results not shown). In all subsequent adhesion assays the water-soluble mucilage was removed by washing spores.

IIF was used to determine at what stage, after incubation of spores on hydrophobic silane-coated glass slides, the adhesive glycoproteins present in the ECMs of germ tubes and appressoria (recognised by MAbs UB26 and UB31) were expressed. Labelling with both UB26 and UB31 was not observed until incubation of spores on slides for a period of $60 \mathrm{~min}$, usually at one end of the conidium (Fig. 2A-D). Labelling of developing germ tubes by UB26 (not shown), and appressoria by UB31 (Fig. $2 \mathrm{E}$ and $\mathrm{F}$ ) was much stronger after $5 \mathrm{~h}$ incubation on slides. At all time points, UB20 strongly and uniformly labelled the surface of all spores (results not shown, but similar to labelling shown previously e.g. by Pain et al. [34]. UBIM22, the negative control antibody, did not label any of the samples.

On the basis of these results, we judged that adhesive ECM glycoproteins recognised by UB26 and UB31 were not released until $1 \mathrm{~h}$ after incubation of spores on slides, so that the initial adhesion of spores observed on polystyrene at $<1 \mathrm{~h}$ (Fig. 1) was not due to this secreted ECM. This was further investigated by assessing adhesion of spores to polystyrene at $30 \mathrm{~min}$ i.e. before ECM secretion. Incubations at low temperature $\left(4^{\circ} \mathrm{C}\right)$ or in the presence of sodium azide, a respiratory pathway inhibitor showed that adhesion was not inhibited (Table 2). Cycloheximide, a protein synthesis inhibitor, caused a small reduction in adhesion, though this was not significant $(P>0.05)$. Azide and cycloheximide treatment inhibited germination and appressorium formation of spores, assessed after a $24 \mathrm{~h}$ incubation, showing that they were having profound effects on spore metabolism (Table 2). 

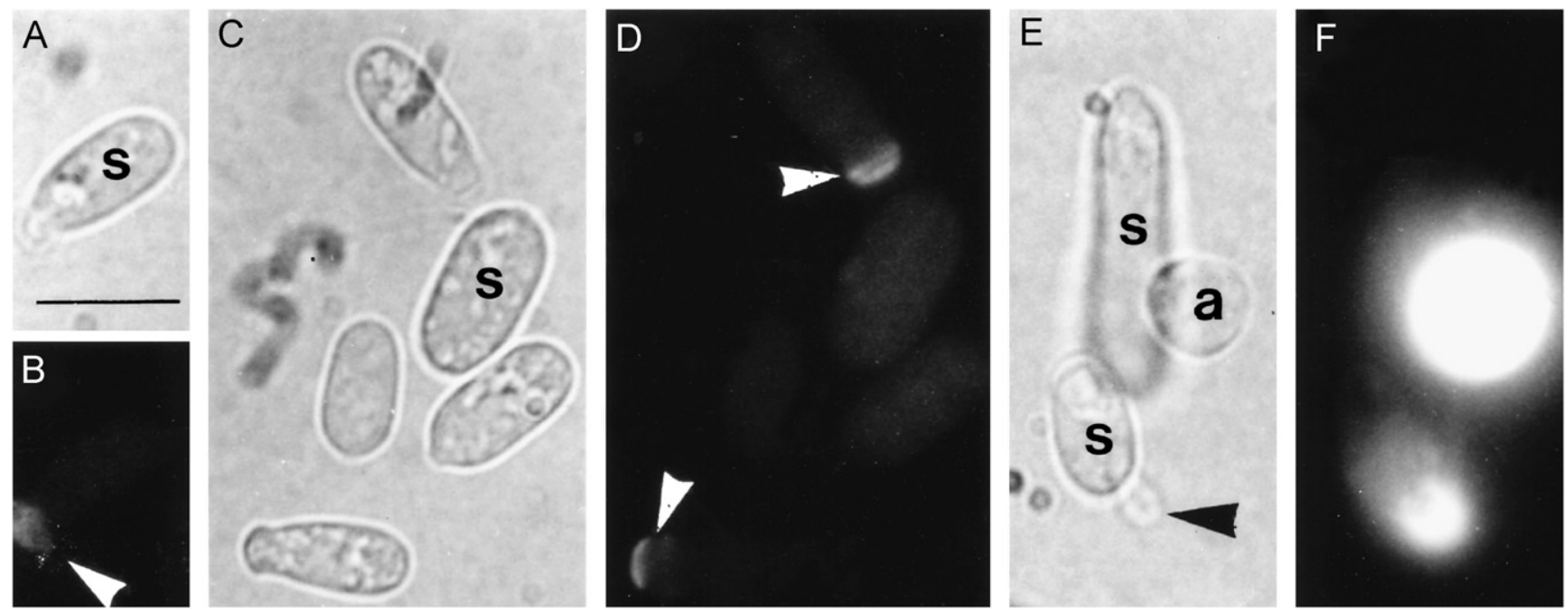

Fig. 2. Immunofluorescence labelling of spores, germ-tubes and appressoria on silanised glass slides. Samples were labelled with MAbs UB26 or UB31 and viewed by DIC (A,C,E) or epi-fluorescence microscopy (B,D,F). Spores were incubated on slides for $1 \mathrm{~h}$ before labelling with UB26 (A,B) or UB31 (C,D). The antibodies labelled one end (arrow heads) of some of the spores, although germ-tubes were not apparent at this stage. After $5 \mathrm{~h}$ incubation of spores (s) on slides, germ-tubes (arrow) were seen and appressoria (a) had developed and these were strongly labelled by UB31, (E,F). Bar $=10 \mu \mathrm{m}$.

Table 2

Effects of various treatments on spore adhesion, germination and appressorium formation

\begin{tabular}{|c|c|c|c|c|c|c|}
\hline Treatment $^{\mathrm{a}}$ & \multicolumn{2}{|c|}{ Adhesion ${ }^{\mathrm{b}}(\%)$} & \multicolumn{2}{|c|}{ Germination $^{\mathrm{b}}(\%)$} & \multicolumn{2}{|c|}{ Appressoria ${ }^{\mathrm{b}}(\%)$} \\
\hline Cycloheximide & $88.2 \pm 7.4$ & $70.3 \pm 6.8$ & $69.6 \pm 9.9$ & 0 & $23.7 \pm 3.9$ & 0 \\
\hline $4^{\circ} \mathrm{C}$ & $86.8 \pm 8.6$ & $92.9 \pm 5.3$ & $60.4 \pm 7.9$ & 0 & $40.6 \pm 7.3$ & 0 \\
\hline
\end{tabular}

${ }^{\text {a }}$ Spores were incubated in $2 \mathrm{mM}$ sodium azide or $25 \mu \mathrm{g} \mathrm{ml} l^{-1}$ cycloheximide or at $4{ }^{\circ} \mathrm{C}$.

${ }^{\mathrm{b}}$ Adhesion was measured on polystyrene after a $30 \mathrm{~min}$ incubation and germination and appressorium formation after $24 \mathrm{~h}$ incubation. Results are the means \pm the standard errors from three replicates.

Table 3

Effects of protease treatment and UB20 treatment of spores on adhesion at $30 \mathrm{~min}$ and $24 \mathrm{~h}$, and on germination and appressorium formation

\begin{tabular}{lclll}
\hline Treatment $^{\mathrm{a}}$ & Adhesion at $30 \min ^{\mathrm{b}}(\%)$ & Adhesion at $24 \mathrm{~h}^{\mathrm{b}}(\%)$ & Germination $^{\mathrm{c}}(\%)$ & Appressoria $^{\mathrm{c}}(\%)$ \\
\hline Control & $89.2 \pm 9.0$ & $79.6 \pm 2.2$ & $38.5 \pm 7.5$ & $29.9 \pm 5.6$ \\
Protease & $13.4 \pm 3.0$ & $91.0 \pm 14.6$ & $27.2 \pm 0.74$ & 0 \\
Inactivated-protease & $92.8 \pm 9.7$ & N.D. & $39.0 \pm 6.0$ & $28.3 \pm 4.6$ \\
UB20 & $21.7 \pm 14.7$ & $84.2 \pm 19.9$ & $35.9 \pm 5.7$ & $32.2 \pm 4.0$ \\
UBIM22 & $100.2 \pm 1.6$ & $94.3 \pm 21.2$ & $46.3 \pm 8.9$ & $35.6 \pm 7.5$ \\
\hline
\end{tabular}

\footnotetext{
${ }^{\mathrm{a}}$ Spores were pre-treated with protease, heat inactivated protease, UB20 or UBIM22. determined.

\subsection{Effects of removal of the spore coat and the MAb UB20 on germination and appressorium formation}

${ }^{\mathrm{b}}$ The $\%$ adhesion was determined after $30 \mathrm{~min}$ and $24 \mathrm{~h}$ incubation on polystyrene dishes. Results are the means \pm of three replicates. N.D: not

${ }^{\mathrm{c}}$ Germination and the percentage of spores which had differentiated appressoria was assessed after $24 \mathrm{~h}$ incubation.

The role of the spore coat in the processes described above was tested by using two treatments. Protease treatment removes the spore coat, but leaves the spore wall intact [35].
The MAb UB20 binds to the major glycoproteins present in the spore coat [35]. Removal of the spore coat with protease treatment reduced germination, but totally inhibited the formation of appressoria (Table 3). However, incubation of spores with the MAb UB20 IgG did not affect either germination or appressorium formation (Table 3). 

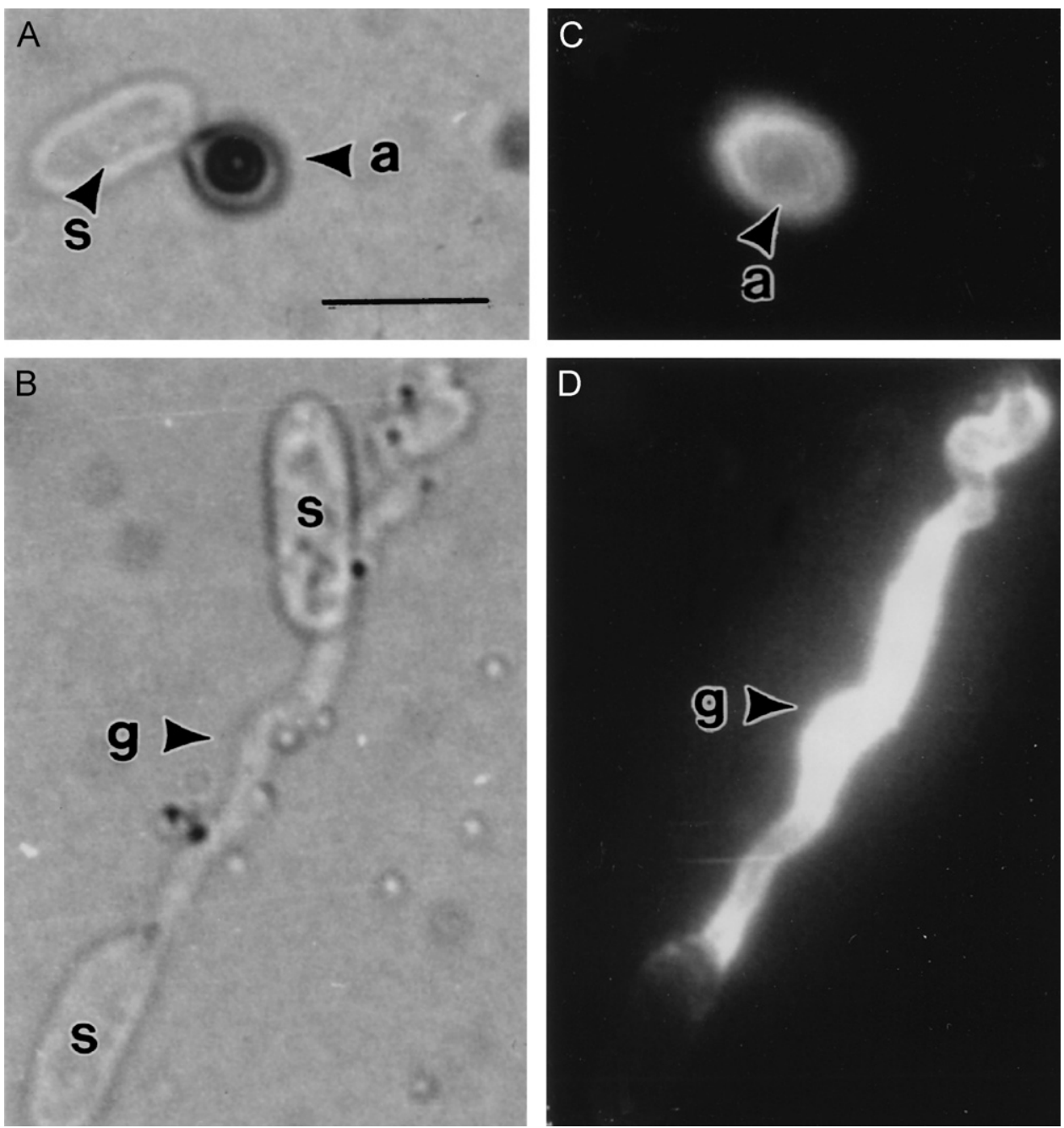

Fig. 3. Immunofluorescence labelling of germinating spores with UB26. Spores and protease-treated spores were incubated on glass slides for $24 \mathrm{~h}$ before labelling with UB26 and viewed by DIC (A,C) or epi-fluorescence microscopy (B,D). UB26 labelled the germ-tubes (not shown) and appressoria (a) that developed from untreated spores $(\mathrm{A}, \mathrm{B})$ and labelled germ-tubes $(\mathrm{g})$ formed by the protease-treated spores $(\mathrm{C}, \mathrm{D})$ ). Appressoria did not form in the latter condition. Bar $=10 \mu \mathrm{m}$.
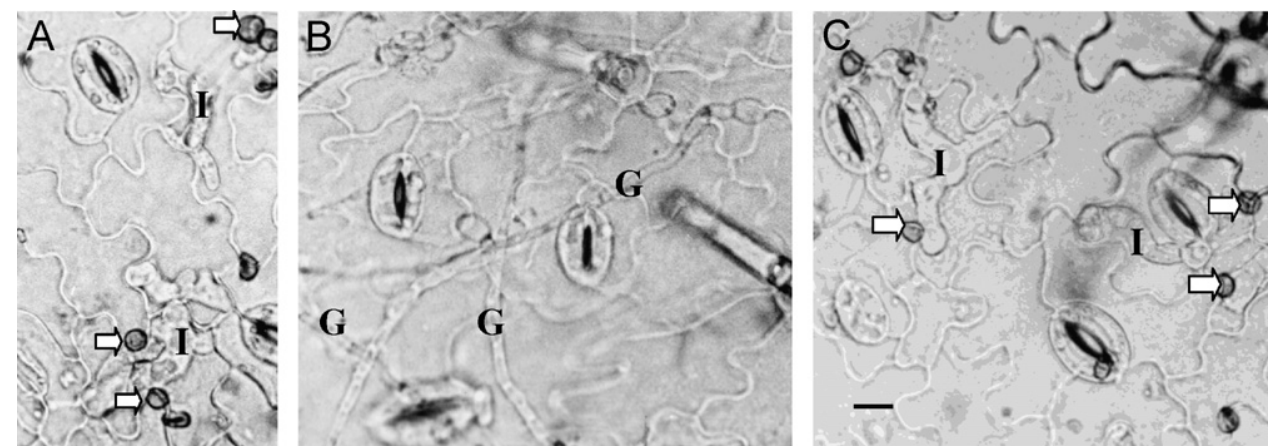

Fig. 4. Epidermal peels from bean leaves inoculated with untreated spores (A), spores pre-treated with protease (B) and spores pre-treated with UB20 (C). Samples were observed by DIC microscopy. Untreated spores and spores pre-treated with UB20 developed appressoria (arrows) and intracellular hyphae (I), however, spores pre-treated with protease formed germ-tubes $(\mathrm{G})$, but did not develop appressoria. Bar $=10 \mu \mathrm{m}$.

\subsection{Effects of removal of the spore coat and the MAb UB20 on adhesion}

Protease-treatment of spores considerably reduced adhesion when compared to that of untreated spores after incubation for $30 \mathrm{~min}$ on polystyrene (Table 3 ). Similar effects were observed after incubation of spores with $\mathrm{MAb}$ UB20, in agreement with a previous report [35]. However, adhesion of spores/germinated spores after $24 \mathrm{~h}$ incubation on slides was unaffected by removal of the spore coat by 
protease treatment or by UB20 (Table 3). In order to determine whether ECM glycoproteins had been secreted by protease-treated spores and germ-tubes that had adhered after $24 \mathrm{~h}$ incubation on glass slides, these structures were labelled with UB26 and observed by IIF. The results showed labelling by this antibody of adhered spores, with germ-tubes emerging from them, although no appressoria had developed (Fig. 3).

\subsection{The role of the spore coat in pathogenicity}

The role of the spore coat in the development of infection structures and pathogenicity of $C$. lindemuthianum on bean leaves was tested by treating spores with protease or the MAb UB20. Protease-treated spores produced no appressoria on leaves and subsequently the fungus could not penetrate the host plant and form intracellular hyphae (Fig. 4). There were no symptoms of infection on leaves (Fig. 5). Coating spores with UB20 did
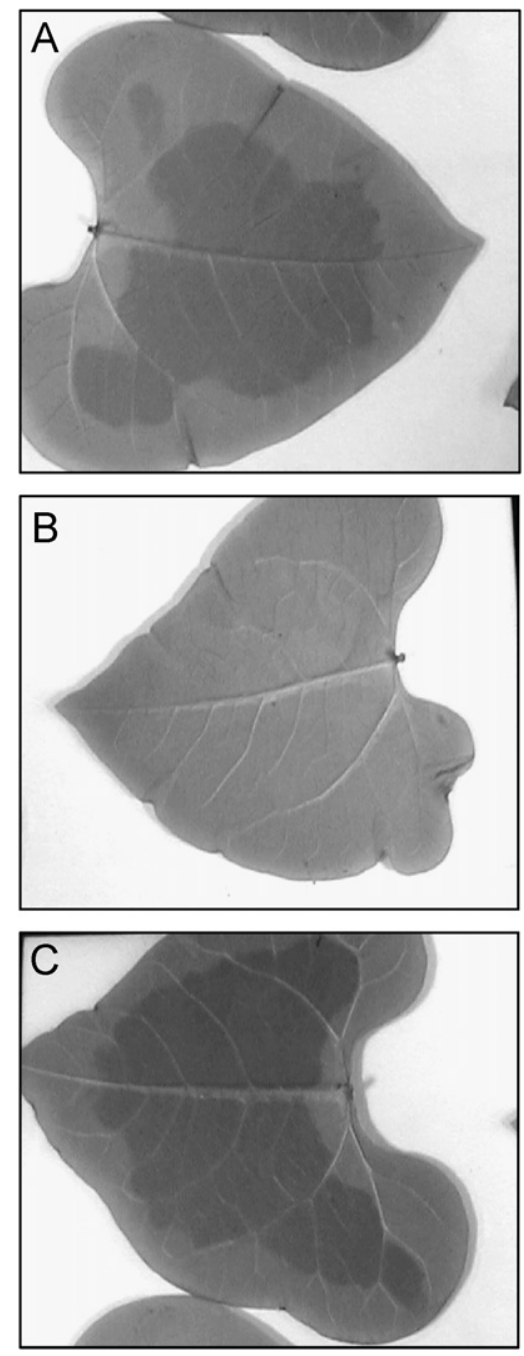

Fig. 5. Pathogenicity tests on excised leaves inoculated with untreated spores (A), spores pre-treated with protease (B) and spores pre-treated with UB20 (C). Leaves were photographed 7 days after inoculation. Dark, spreading lesions were visible on the leaf surfaces shown in A and C. not affect pathogenicity on bean leaves (Fig. 5) and there was no effect of the antibody on appressorium formation (Fig. 4).

\section{Discussion}

\subsection{C. lindemuthianum spores adhere to a hydrophobic surface before the release of ECM glycoproteins that consolidate adhesion}

The evidence presented in this paper shows that adhesion in $C$. lindemuthianum is a two-stage process beginning with the initial passive adhesion of the spore to a hydrophobic surface, followed by consolidation of adhesion with the secretion of ECM glycoproteins prior to, and during, germination. The evidence for this is as follows. In previous studies, high molecular weight ECM glycoproteins with adhesive properties, surrounding germ-tubes and appressoria of germinating $C$. lindemuthianum spores, have been identified with the MAbs UB26 and UB31 [32,36,37]. In the present work, we have shown that these antibodies label the apices of spores, as early as $1 \mathrm{~h}$ after incubation of spores on a substratum, and before observable germination. These antibodies also labelled the emergent germtubes and differentiating appressoria.

However, in adhesion assays, C. lindemuthianum spores clearly adhered to a hydrophobic, polystyrene surface as early as $10 \mathrm{~min}$ after settlement, well before germination and the secretion of ECM. The evidence obtained with metabolic inhibitors and the use of incubations at low temperature suggests that metabolic activity is not required for the initial adhesion of spores to a hydrophobic surface, i.e. before ECM secretion. Protease treatment of spores inhibited adhesion to polystyrene and, since the protease was washed off the spores before the assay, this provides further evidence to suggest that there is no release of adhesive material once the spores are applied to the slides. Spores did not adhere rapidly to the surface of hydrophilic glass, but adhesion to glass gradually increased concomitantly with the secretion of the adhesive ECM glycoproteins recognised by UB26 and UB31.

The results of sodium azide and cycloheximide treatment of spores are similar to findings on the maize anthracnose pathogen C. graminicola [22]. However, the results with azide are contrary to previously published findings on C. lindemuthianum adhesion [42]. The discrepancy may result from the fact that these authors measured adhesion at $1 \mathrm{~h}$ incubation, when secretion of ECM proteins is taking place (see above).

Adhesion of $C$. lindemuthianum spores is similar to that reported for several other fungi e.g. Botrytis cinerea, Discula umbrinella, Phyllosticta ampelicida and Bipolaris sorokiniana, in which there is an initial phase of adhesion mediated by hydrophobic interactions before the secretion of an adhesive ECM [7,9,29,43,44]. This mechanism of adhesion is clearly different to that of some other fungal pathogens in which pre-formed adhesive is secreted 
on contact with a surface e.g. M. grisea [26] and $N$. haematococca [45].

The polystyrene surface has similar hydrophobicity to bean hypocotyls [35] and this suggests that the adhesion of C. lindemuthianum spores mediated by these early interactions is an important part of the normal infection process to attach the fungal spores to the plant host [20].

\subsection{Appressorium formation requires spore contact with a hard surface but does not require spore adhesion mediated by hydrophobic interactions}

The results showed that spores of $C$. lindemuthianum germinated on glass and polystyrene, but less well on water agar, whereas appressoria only developed on glass and polystyrene. Therefore spore surface contact with a hard surface is required for appressorium formation, but this surface does not need to be hydrophobic. Furthermore, an initial phase of passive adhesion of spores to a hydrophobic surface is not required for appressorium formation since appressoria developed on glass. These findings therefore suggest that rapid, passive adhesion of spores to a hydrophobic surface and contact with a hard surface leading to appressorium formation are independent events.

Results on other Colletotrichum species, including C. gloeosporioides [13], C. trifolii [5], C. acutatum [46] and C. graminicola [8] have also shown that contact with a hard surface is required for appressorium formation. Most fungi will germinate without a requirement for contact with a hard surface, although some fungi, e.g. Phyllosticta species and C. graminicola require initial attachment for germination as well as appressorium formation $[7-9,17]$.

\subsection{The spore coat is required for rapid adhesion of spores to a hydrophobic surface but is not required for the subsequent secretion of ECM glycoproteins}

Previous work has shown that the MAb UB20, which recognises several spore coat proteins of $C$. lindemuthianum, inhibits adhesion when added to spores [35] and this was confirmed in the present study. Treatment of spores with a protease that removes the spore coat significantly reduced adhesion when compared to control spores, providing further evidence that the spore coat mediates the initial adhesion in C. lindemuthianum. In addition, since the protease was removed prior to the assay, this shows that adhesion depends only on the pre-formed spore coat and not on any released material. However, neither protease nor UB20 treatment of spores prevented adhesion when assessed at $24 \mathrm{~h}$, and neither treatment blocked the secretion of ECM glycoproteins. Thus, the spore coat is not required for interactions with a substratum that leads to this second phase of adhesion.

Evidence from TEM studies on D. umbrinella and $P$. ampellicida spores shows that, like $C$. lindemuthianum, they have a distinctive extra layer, or sheath, outside of the wall; this layer is rich in glycoproteins, but lacks the polysaccharides characteristic of fungal walls [9,29]. A further similarity with $C$. lindemuthianum is that removal of the outer layers of glycoproteins with protease also inhibits spore adhesion in these two fungi $[9,29]$.

\subsection{An intact spore coat is required for appressorium formation}

Removal of the $C$. lindemuthianum spore coat with protease treatment did not affect germination, but inhibited appressorium formation. However, spores incubated with the MAb UB20 were still able to differentiate appressoria. This suggests that an intact spore coat is needed for spores to respond to interactions with a hard surface that lead to the formation of appressoria. Coverage of spore coat glycoproteins with an antibody is clearly not sufficient to disrupt these interactions. Since incubation of spores with the MAb UB20 inhibited the initial passive adhesion of spores to polystyrene, this supports the view that this rapid adhesion to a hydrophobic surface is not required for appressorium formation.

For C. graminicola, contact and/or adhesion of germtubes to a substratum is needed for appressorium formation, and it has been shown that $>4 \mu \mathrm{m}$ of continuous contact of the germ-tubes with a hydrophobic substratum is required in this case [7]. However, protease-treated spores of $C$. lindemuthianum still germinated and produced adhesive ECM glycoproteins, which suggests that these processes are not sufficient to trigger appressorium formation without the early contact of the spore with a hard surface.

\subsection{The spore coat and pathogenicity}

Treatment of $C$. lindemuthianum spores with protease and UB20 produced similar effects on appressorium formation on inoculated bean leaves compared to those obtained on artificial surfaces. Protease treatment led to the inhibition of appressorium formation and the fungus was not able to penetrate leaves, leading to a reduction in pathogenicity. In contrast, UB20 treatment had no effect on appressorium formation and no effect on pathogenicity. Thus an intact spore coat is required for pathogenicity. In some assays we attempted to dislodge spores from leaves by various washing procedures, in order to assess adhesion, but this yielded inconsistent results. Therefore, the assays reported involved the use of inoculated leaves which were not disturbed and it was not possible to test directly whether the treatments were affecting adhesion of spores to the leaf surface. However, results on $C$. graminicola have shown that spore adhesion is an important factor in the progression of pathogenicity on maize leaves [20]. Clearly, adhesion of ungerminated spores ensures they are not displaced from the leaf either by wind, rainfall or by the inoculum rolling off the leaf. 


\subsection{The spore coat is involved in two separate functions}

The evidence presented in this paper shows that the spore coat of $C$. lindemthianum has at least two functions; it is necessary for adhesion of spores to a hydrophobic surface and it is required as part of the mechanisms involved in interactions with a hard surface to initiate the differentiation of appressoria. The composition of the spore coat is relatively simple, since it is made up of several glycoproteins [35]. Protease treatment removes the spore coat and its effects have demonstrated that an intact structure is required for both adhesion and triggering appressorium formation. UB20 binds to the major glycoproteins in the spore coat, including the $110 \mathrm{kDa}$ hydrophobic protein, and the antibody could be blocking spore adhesion by masking this protein. The fact that UB20 binding to spores has no effect on appressorium formation may suggest that covering the spore surface is not sufficient to block the perception by spores of a signal on making contact with a hard surface. This interaction may involve fungal cell wall and/or plasma membrane deformation and this could explain why appressoria do not form when spores are incubated on a soft substratum, such as water agar, or in liquid culture. It has also been suggested that flattening of the spore wall or germ-tube could result in stretching or tension in the plasma membrane that could lead to the opening of mechanosensitive ion channels [7]. Overall, the results reported in this paper, taken together with published results, show that interactions of spores with the substratum by different pathogenic fungal species, and also within Colletotrichum species, are remarkably varied.

\section{Acknowledgements}

This work was supported by a BBSRC Special CASE studentship to $\mathrm{S}$. Rawlings and was carried out under authority of DEFRA licence PHF 870B/405/33.

\section{References}

[1] Xiao JZ, Watanabe T, Kamakura T, Ohshima A, Yamaguchi I. Studies on cellular differentiation of Magnaporthe grisea. Physicochemical aspects of substratum surfaces in relation to appressorium formation. Physiol Mol Plant Pathol 1994;44:227-36.

[2] Doehlemann G, Berndt P, Hahn M. Different signalling pathways involving a $\mathrm{G} \alpha$ protein, cAMP and a MAP kinase control germination of Botrytis cinerea conidia. Mol Microbiol 2006;59: 821-35.

[3] Ahn IP, Uhm KH, Kim S, Lee Y-H. Signaling pathways involved in preinfection development of Colletotrichum gloeosporioides, C. coccodes, and C. dematium pathogenic on red pepper. Physiol Mol Plant Pathol 2003;63:281-9.

[4] Kim Y, Liu Z, Daoxin L, Kollatukudy P. Two novel genes induced by hard-surface contact of Colletotrichum gloeosporioides conidia. J Bacteriol 2000;182:4688-95.

[5] Buhr TL, Dickman MB. Gene expression analysis during conidial germ tube and appressorium development in Colletotrichum trifolii. Appl Environ Microbiol 1997;63:2378-83.
[6] Takano Y, Kikuchi T, Kubo Y, Hamer JE, Kazuyukiv M, Furusawa I. The Colletotrichum lagenarium MAP kinase gene CMK1 regulates diverse aspects of fungal pathogenesis. Mol Plant Microbe Int 2000;13:274-83.

[7] Apoga D, Barnard J, Craighead HG, Hoch HC. Quantification of substratum contact required for initiation of Colletotrichum graminicola appressoria. Fungal Gen Biol 2004;41:1-12.

[8] Chaky J, Anderson K, Moss M, Vaillancourt L. Surface hydrophobicity and surface rigidity induce spore germination in Colletotrichum graminicola. Phytopathol 2001;91:558-64.

[9] Shaw BD, Hoch HC. The pycnidiospore of Phyllosticta ampelicida: surface properties involved in substratum attachment and germination. Mycol Res 1999;103:915-24.

[10] Podila GP, Rogers LM, Kolattukudy P. Chemical signals from avocado surface wax trigger germination and appressorium formation in Colletotrichum gloeosporioides. Plant Physiol 1993;103:267-72.

[11] Dickman MB, Ha YS, Yang Z, Adams B, Huang C. A protein kinase from Colletotrichum trifolii is induced by plant cutin and is required for appressorium formation. Mol Plant Microbe Int 2003;16:411-21.

[12] Kim Y, Wang Y, Liu Z, Kolattukudy P. Identification of a hard surface contact-induced gene in Colletotrichum gloeosporioides conidia as a sterol glycosyl transferase, a novel fungal virulence factor. Plant J 2002;30:177-87.

[13] Liu Z-M, Kolattukudy P. Identification of a gene product induced by hard-surface contact of Colletotrichum gloeosprioides conidia as a ubiquitin-conjugating enzyme by yeast complementation. J Bacteriol 1998;180:3592-7.

[14] Liu Z-M, Kolattukudy P. Early expression of the calmodulin gene, which precedes appressorium formation in Magnaporthe grisea, is inhibited by self-inhibitors and requires surface attachment. J Bacteriol 1999;181:3571-7.

[15] Yang Z, Dickman MB. Regulation of cAMP and cAMP dependent protein kinase during conidial germination and appressorium formation in Colletotrichum trifolii. Physiol Mol Plant Pathol 1997; $12: 430-9$.

[16] Kuo K, Hoch HC. Germination of Phyllosticta ampelicida pycnidiospores: prerequisite of adhesion to the substratum and the relationship of substratum wettability. Fungal Gen Biol 1996;20: $18-29$.

[17] Shaw BD, Carroll GC, Hoch HC. Generality of the prerequisite of conidium attachment to a hydrophobic substratum as a signal for germination among Phyllosticta species. Mycologia 2006;98:186-94.

[18] Warwar V, Dickman MB. Effects of calcium and calmodulin on spore germination and cappressoirum development in Colletotrichum trifolii. Appl Environ Microbiol 1996;62:74-9.

[19] Xiao J, Ohshima A, Kamakur T, Ishiyama T, Yamaguchi I. Extracellular glycoproteins associated with cellular differentiation in Magnaporthe grisea. Mol Plant Microbe Int 1994;7:639-44.

[20] Mercure EW, Kunoh H, Nicholson RL. Adhesion of Colletotrichum graminicola conidia to corn leaves. A requirement for disease development. Physiol Mol Plant Pathol 1994;45:407-20.

[21] Mercure EW, Kunoh H, Nicholson RL. Visualisation of materials released from adhered, ungerminated conidia of Colletotrichum graminicola. Physiol Mol Plant Pathol 1995;46:121-35.

[22] Mercure EW, Leite B, Nicholson RL. Adhesion of ungerminated conidia of Colletotrichum graminicola to artificial hydrophobic surfaces. Physiol Mol Plant Pathol 1994;45:421-40.

[23] Sela-Buurlage MB, Epstein L, Rodriguez RJ. Adhesion of ungerminated Colletotrichum musae conidia. Physiol Mol Plant Pathol 1991; 39:345-52.

[24] Kunoh H, Yamaoka N, Yoshioka H, Nicholson RL. Preparation of the infection court by Erysiphe graminis I. Contact-mediated changes in the morphology of the conidium surface. Exp Mycol 1988;12: 325-35.

[25] Wright AJ, Thomas BJ, Kunoh H, Nicholson RL, Carver TLW. Influences of substrata and interface geometry on the release of extracellular material by Blumeria graminis conidia. Physiol Mol Plant Pathol 2002;61:163-78. 
[26] Hamer JE, Howard RJ, Chumley FG, Valent B. A mechanism for surface attachment of spores of a plant pathogenic fungus. Science 1987;239:288-90.

[27] Ahn N, Kim S, Choi W, Im K-H, Lee Y-H. Extracellular matrix protein gene, EMP1, is required for appressorium formation and pathogenicity of the rice blast fungus Magnaporthe grisea. Mol Cells 2004; 17:166-73.

[28] Robold AV, Hardham AR. During attachment Phytophthora spores secrete proteins containing thrombospondin type 1 repeats. Curr Gen $2005 ; 47: 307-15$

[29] Viret O, Toti L, Chapela IH, Petrini O. The role of the extracellular sheath in recognition and attachment of conidia of Discula umbrinella (Berk. \& Br.) Morelet to the host surface. New Phytol 1994;127:123-31.

[30] Bailey JA, O'Connell RJ, Pring RJ, Nash C. Infection strategies of Colletotrichum species. In: Bailey JA, Jeger MJ, editors. Colletotrichum: biology, pathology and control. Wallingford: CAB International; 1992. p. 88-120.

[31] Perfect SE, Hughes HB, O'Connell RJ, Green JR. Colletotrichum: a model genus for studies on pathology and fungal-plant interaction. Fungal Gen Biol 1999;27:186-98.

[32] O'Connell RJ, Pain NA, Hutchison KA, Jones GL, Green JR. Ultrastructure and composition of the cell surfaces of infection structures formed by the fungal plant pathogen Colletotrichum lindemuthianum. J Microsc 1996;181:204-12.

[33] Van Dyke CG, Mims CW. Ultrastructure of conidia, conidium germination and appresorium development in the plant pathogenic fungus Colletotrichum truncatum. Can J Bot 1991;69: 2455-67.

[34] Pain NA, O'Connell RJ, Bailey JA, Green JR. Monoclonal antibodies which show restricted binding to four Colletotrichum species: C. lindemuthianum, C. malvarum, C. orbiculare and C. trifolii. Physiol Mol Plant Pathol 1992;40:111-26.

[35] Hughes HB, Carzaniga R, Rawlings SL, Green JR, O'Connell RJ. Spore surface glycoproteins of Colletotrichum lindemuthianum are recognised by a monoclonal antibody which inhibits adhesion to polystyrene. Microbiology 1999;145:1927-36.

[36] Hutchison KA, Green JR, Wharton PS, O'Connell RJ. Identification and localisation of glycoproteins in the extracellular matrices around germ-tubes and appressoria of Colletotrichum species. Mycol Res 2002;106:729-36.

[37] Pain NA, Green JR, Jones GL, O'Connell RJ. Composition and organisation of extracellular matrices around germ tubes and appressoria of Colletotrichum lindemuthianum. Protoplasma 1996; 190:119-30.

[38] Mathur RS, Barnett HL, Lilly VG. Sporulation of Colletotrichum lindemuthianum in culture. Phytopathol 1950;40:104-14.

[39] Leite B, Nicholson RL. Mycosporine-alanine: a self-inhibitor of germination from the conidial mucilage of Colletotrichum graminicola. Exp Mycol 1992;16:76-86.

[40] Perry J, Gilligan M, Green E, Docherty H, Heath D. Monoclonal antibodies to ROS 17/2.8 cells recognise antigens, some of which are restricted to osteoblasts and chondrocytes. J Bone Min Res 1990;5:187-200.

[41] Harlow E, Lane D. Antibodies: a laboratory manual. New York, USA: Cold Spring Harbour Laboratories; 1988.

[42] Young DH, Kauss H. Adhesion of Colletotrichum lindemuthianum spores to Phaseolus vulgaris hypocotyls and to polystyrene. Appl Environ Microbiol 1984;47:616-9.

[43] Apoga D, Jansson HB, Tunlid A. Adhesion of conidia and germlings of the plant pathogenic fungus Bipolaris sorokiniana to solid surfaces. Mycol Res 2001;105:1251-60.

[44] Doss RP, Potter SW, Chastagnen GA, Christian JK. Adhesion of non-germinated Botrytis cinerea conidia to several substrata. Appl Environ Microbiol 1993;59:1786-91.

[45] Kwon YH, Epstein L. Involvement of the $90 \mathrm{kDa}$ glycoprotein in adhesion of Nectria haematococca macroconidia. Physiol Mol Plant Pathol 1997;51:287-303.

[46] Egley GH. Substrate surface influences upon germination of Colletotrichum truncatum conidia. Can J Bot 1994;72:1758-65. 